\title{
Clinical Study of 107 Hospitalized Patients with Peritonsillar Abscess
}

\author{
Risa Wakisaka ${ }^{1}$ Takumi Kumai ${ }^{1,2}$ Kan Kishibe ${ }^{1}$ Miki Takahara ${ }^{1}$ Akihiro Katada ${ }^{1}$ Tatsuya Hayashi ${ }^{1,2}$ \\ Yasuaki Harabuchi ${ }^{1}$ \\ ${ }^{1}$ Department of Otolaryngology-Head and Neck Surgery, Asahikawa \\ Medical University, Hokkaido, Japan \\ 2 Department of Innovative Head and Neck Cancer Research and \\ Treatment (IHNCRT), Asahikawa Medical University, Hokkaido, Japan \\ Address for correspondence Risa Wakisaka, MD, Department of \\ Otolaryngology-Head and Neck Surgery, Asahikawa Medical University, \\ 1-chōme-1-1 Higashi 2-jō Asahikawa, Hokkaido 078-8510, Japan \\ (e-mail: r-wakisaka@asahikawa-med.ac.jp).
}

Int J Pract Otolaryngol 2021;4:e11-e16.

\begin{abstract}
Keywords

- peritonsillar abscess

- laryngeal edema

- recurrence
\end{abstract}

\section{Introduction}

Peritonsillar abscess occurs when a tonsillar inflammation spreads beyond the tonsillar capsule, resulting in retention of the abscess between the palatine tonsillar capsule and pharyngeal constrictor muscle. It is an illness often encountered in daily otorhinolaryngology. However, when the abscess progresses downward and is complicated with laryngeal edema and deep neck abscesses, peritonsillar abscess can be fatal; hence, appropriate antibiotic therapy and pus drainage are necessary.

We investigated clinical features of 107 cases of peritonsillar abscess that were hospitalized and treated at our department, which are reported herein.

received

May 19, 2020

accepted

August 13, 2020

\section{Subjects and Methods}

Subjects included 107 inpatients with peritonsillar abscess that were treated at our department between January 2014 and December 2018.

The affected site was classified using computed tomography (CT) imaging following the method of Kawabata et al, ${ }^{1}$ where the distance between the hard palate and epiglottic vallecula is divided into superior and inferior poles; it is classified as the superior type when the largest site of abscess was located in the superior pole, and as the inferior type when it was located in the inferior pole. Cases that developed deep neck abscess and other serious complications during

\footnotetext{
(C) 2021. The Author(s).

This is an open access article published by Thieme under the terms of the Creative Commons Attribution-NonDerivative-NonCommercial-License, permitting copying and reproduction so long as the original work is given appropriate credit. Contents may not be used for commercial purposes, or adapted, remixed, transformed or built upon. (https://creativecommons.org/ licenses/by-nc-nd/4.0/) Georg Thieme Verlag KG, Rüdigerstraße 14, 70469 Stuttgart, Germany
} 
hospital stay were excluded from the analysis because they required a different treatment modality.

At our department, the treatment plan is determined by identifying the abscess site on $\mathrm{CT}$, performing paracentesis and incisional drainage, and then administering a penicillin drug (ampicillin/sulbactam [ABPC/SBT]; $3 \mathrm{~g} \times 3$ times daily by intravenous infusion) monotherapy. In the event of any epiglottic edema or arytenoid edema, even if it is mild, hydrocortisone succinate is administered intravenously at an initial dose of $200 \mathrm{mg}$, followed by $100 \mathrm{mg}$ for the subsequent doses, once daily, which are added until the laryngeal edema disappears. The site of paracentesis for superior pole abscesses was located by finding the abscess on CT by reference to the Chiari and Thompson points identified on CT. Pus drainage by paracentesis is performed for inferior pole abscesses only if an oral approach is possible. Pus drained by paracentesis is subjected to a culture test using a sterile swab or anaerobic porter. While pus is expelled, nasal dressing forceps may be used to widen the incision site to continue drainage. The antibiotic's efficacy is assessed on day 3. If the treatment's efficacy is poor, an antibiotic switch can be considered based on the culture results. Steroids were administered for laryngeal edema according to laryngeal endoscopy findings of acute epiglottitis classified I or higher according to the criteria given by Katori and Tsukuda. ${ }^{2}$ Recurrent cases or recurrent tonsillitis are recommended an elective tonsillectomy.

The present study investigated age, sex, affected site, abscess localization, laryngeal edema, tracheostomy, complications, identified bacteria, and drug-resistant bacteria. The relationship between localization and rate of laryngeal edema was analyzed using the chi-squared test and that between localization and length of hospital stay was analyzed using the Student's $t$-test. The relationship between white blood cell at the initial examination and C-reactive protein (CRP) levels was analyzed using the Mann-Whitney U-test. Statistical significance was considered as $p<0.05$.

\section{Results}

\section{Age and Sex}

The cases included 71 males (66\%) and 36 females (34\%), aged 18 to 88 years, with a median age of 44 years. The largest proportion of patients was in their $40 \mathrm{~s}(n=29,27 \%)$, followed by patients in their 30s $(n=19,18 \%$; - Fig. 1$)$.

\section{Affected Site and Localization}

The affected side was the left in 55 patients (51\%), the right in $50(47 \%)$, and bilateral in $2(2 \%)$. The abscess was localized in the superior pole in 71 patients (66\%) and in the inferior pole in 36 (34\%). The mean length of hospital stay among patients with superior and inferior pole abscesses was 6.4 and 7.2 days, respectively; however, the difference in length of hospital stay between patients with different localizations was not significant ( - Fig. 2, $p=0.12$ ). In terms of inflammation parameters on blood tests performed at admission, there was no significant difference in white blood cell count on admission between the patients with different localizations $(p=0.41)$, but CRP levels were significantly higher in patients with inferior pole abscesses (-Fig. 3, $p=0.005$ ).

\section{Medical History}

A total of 19 patients had previous tonsillitis (18\%) and 11 had comorbid diabetes (10\%).

\section{Extent of Inflammation}

A total 35 cases (33\%) were complicated with laryngeal edema, among which 22 (63\%) were inferior pole abscesses,

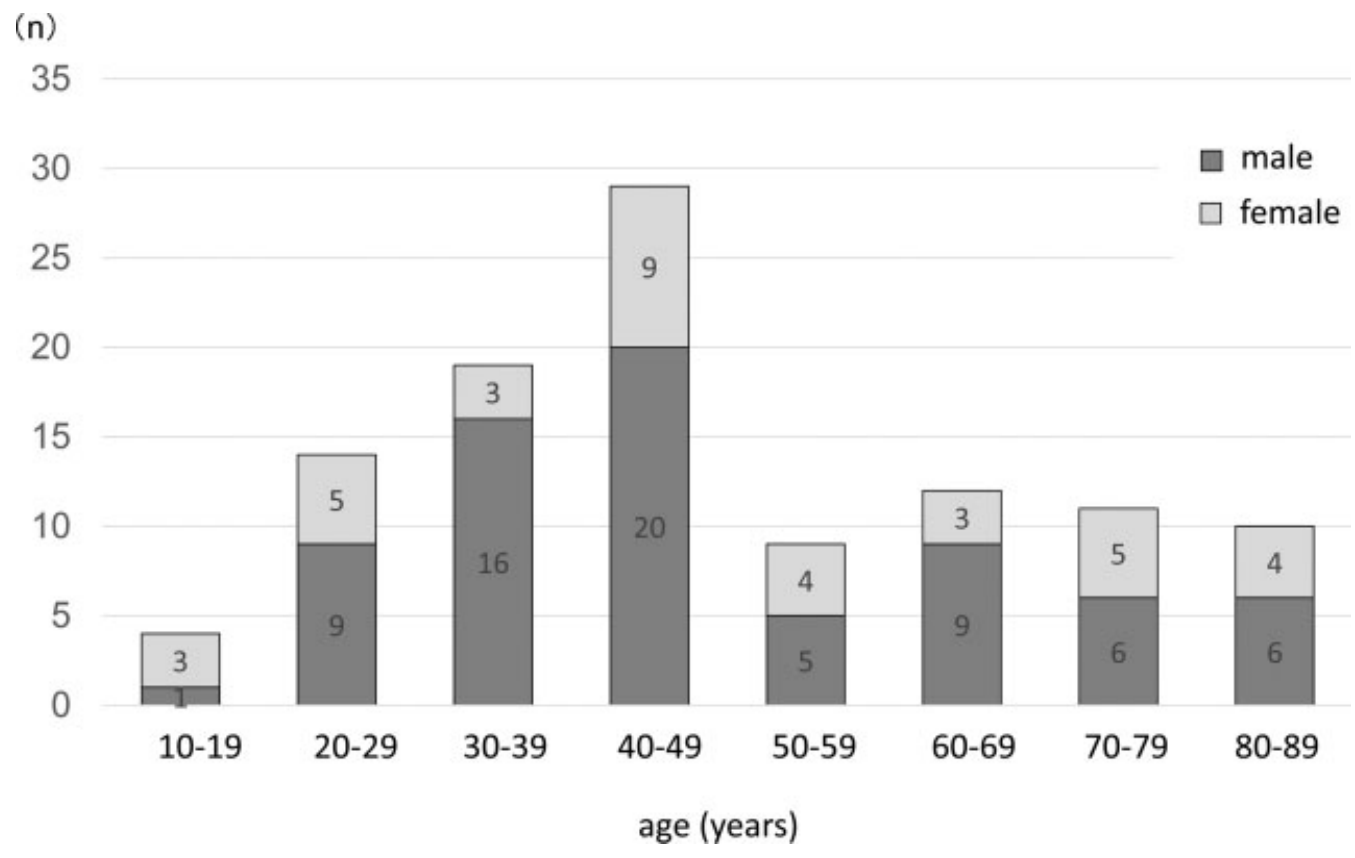

Fig. 1 Subjects (age and sex). The median age was 44 years, and most patients were in their 40 s $(n=29)$. 


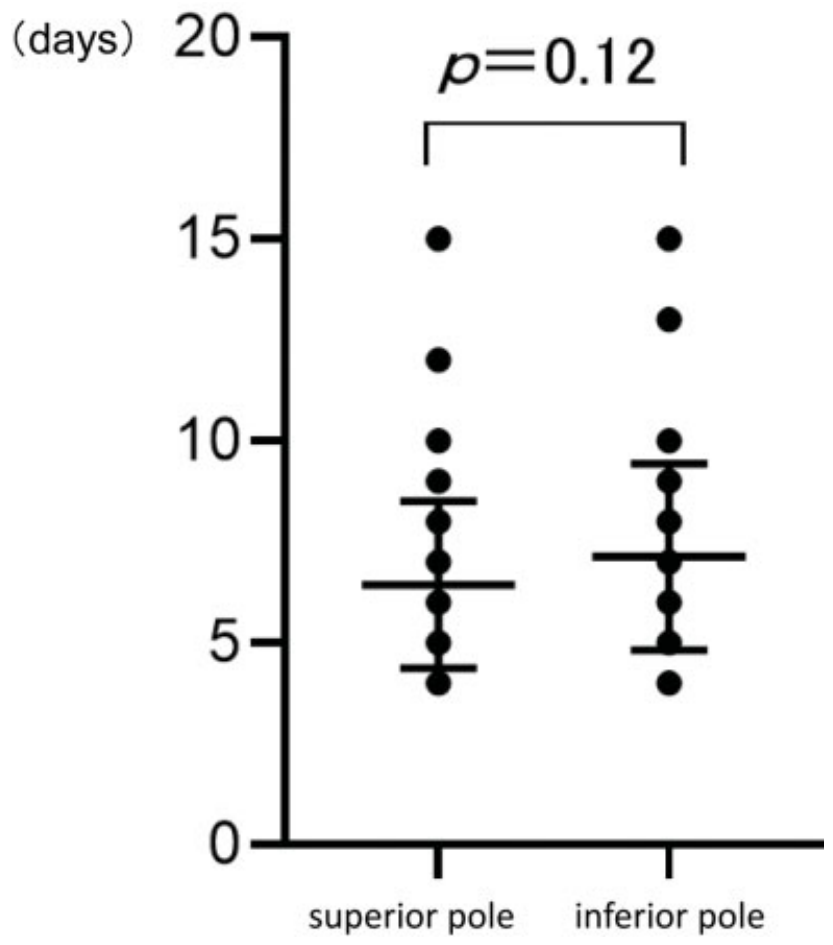

Fig. 2 Hospital stays. The mean hospital stay was 6.4 days. The mean hospital stay for cases of inferior pole abscesses was 7.2 days; there was no significant difference between hospital stays of cases with different abscess sites.

that is, the rate of laryngeal edema complication was significantly higher in cases of inferior pole abscesses (- Fig. 4, $p<0.05$ ). Furthermore, three of the cases complicated with laryngeal edema required a tracheostomy (9\%), all of which had inferior pole abscess.

\section{Treatment Modality}

Incisional drainage was performed at the time of admission on 80 patients (75\%), paracentesis drainage with an injection needle was performed on 21 patients (19\%), and antibiotic therapy was used exclusively for treating six patients (7\%).

Antibiotics were administered in all patients, which included ABPC/SBT monotherapy in 103 patients (96\%). As the symptoms of four patients (4\%) undergoing $\mathrm{ABPC} / \mathrm{SBT}$ monotherapy for 3 days did not improve well, they were switched to meropenem thereafter. Hydrocortisone succinate was used in 43 patients (40\%). Steroid therapy did not shorten the length of hospital stay significantly, as the mean length of hospital stay in patients administered steroids was 6.8 days, compared with 6.5 days for patients who were not administered steroids $(p=0.48)$.

\section{Detected Bacteria and Drug Sensitivity}

Of the 64 patients whose swab cultures were analyzed, aerobic bacteria were detected in the cultures of 36 patients (56\%), and only anaerobic bacteria were detected in the cultures of six patients (11\%). Additionally, 17 patients (25\%) had a mixed infection of aerobic and anaerobic bacteria, one patient had a fungal infection (2\%), and four patients had negative cultures (6\%; - Fig. 5). Among the 74 bacterial strains detected, 18 consisted of the aerobic bacteria $\alpha$-streptococci (24\%). Among the anaerobic bacteria, Fusobacteria were the most common, accounting for nine strains (12\%; - Fig. 6). Drug-resistant bacteria were detected in the cultures of 20 patients (31\%), among which 12 strains were resistant to clarithromycin and erythromycin (39\%, 32\%), 10 strains were resistant to benzylpenicillin and ABPC (18\%), 9 strains were resistant to clindamycin (CLDM; 17\%), and 1 strain was resistant to levofloxacin (3\%). Of the strains with known levels of sensitivity to various antibiotics, the rates of sensitivity are presented in - Fig. $\mathbf{7}$. None of the strains were resistant to ABPC/SBT, which is the first-line therapy used in our department.

\section{Recurrent Cases}

Recurrence was observed in 15 patients (14\%), of which 5 were aged 10 to 29 years (33\%), 7 were aged 30 to 39 years
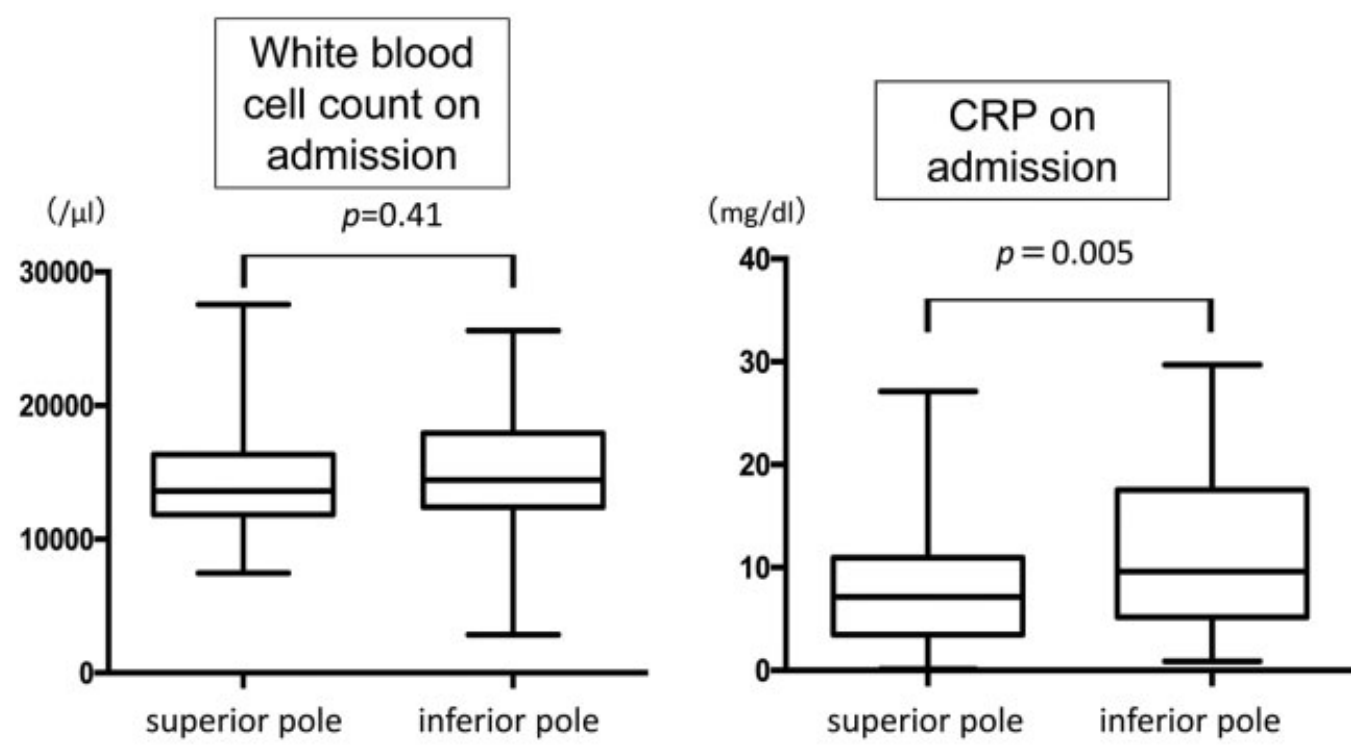

Fig. 3 White blood cell and CRP levels on admission. There was no significant difference in white blood cell count on admission, but CRP levels were significantly higher in cases of inferior pole abscesses. CRP, C-reactive protein. 


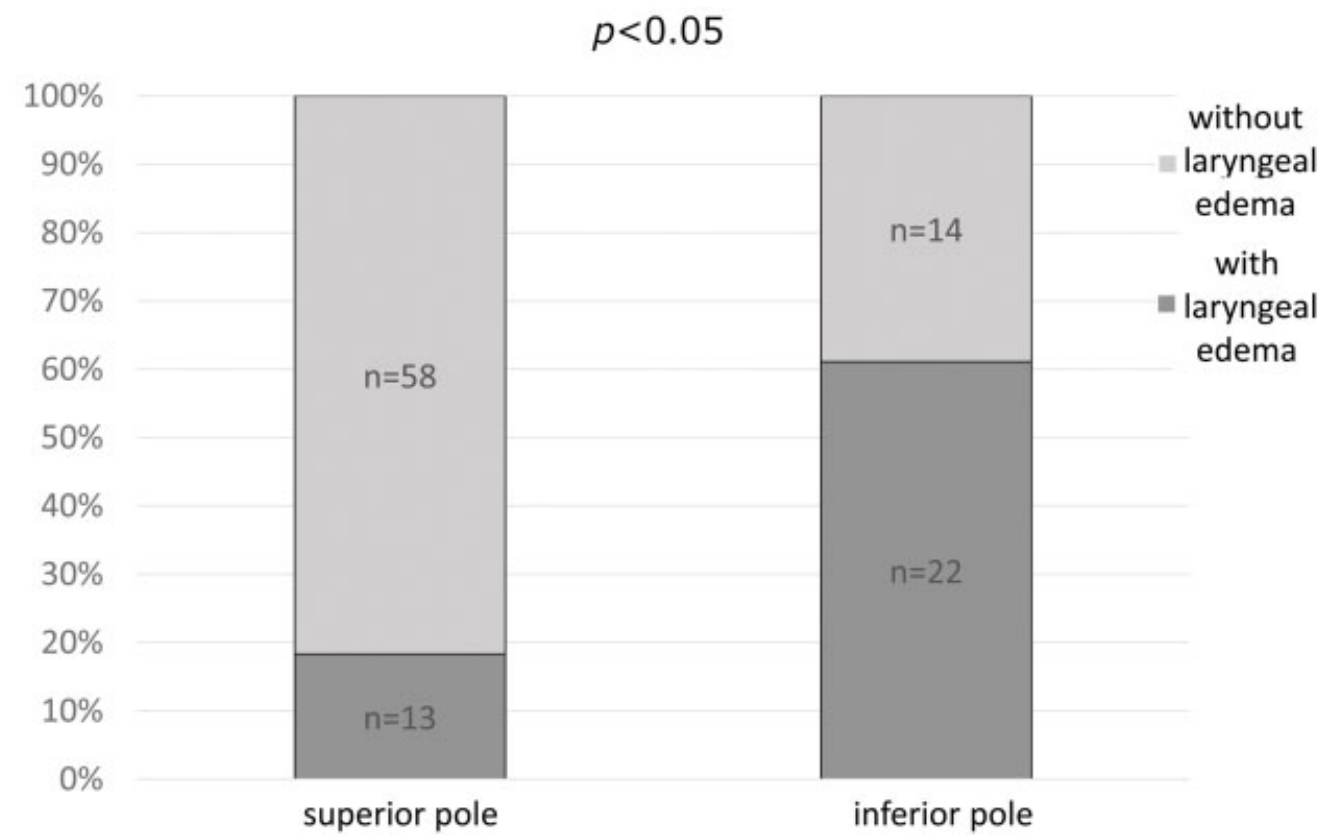

Fig. 4 Rate of abscesses complicated with laryngeal edema according to localization. Laryngeal edema was observed in 35 patients. Of these, 22 patients had abscess localized in the inferior pole, which was significantly higher.

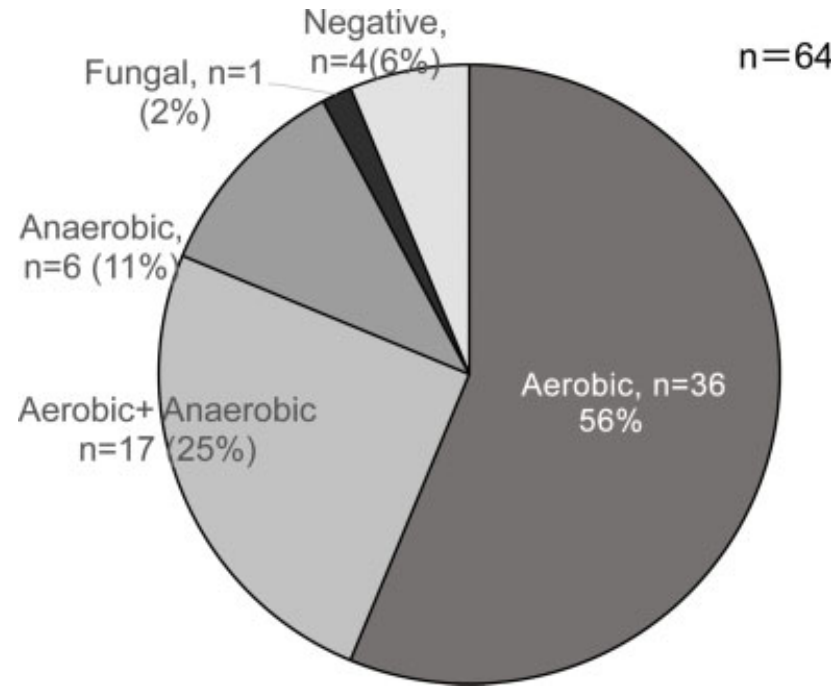

Fig. 5 Detected bacteria $(n=64)$. Only aerobic bacteria were detected in the cultures of 36 patients; only anaerobic bacteria were detected in 6 patients, and a mixed infection of aerobic and anaerobic bacteria was detected in 17 patients.

(47\%), and 3 were aged 50 to 79 years (20\%). Of the recurrent cases, none had comorbid diabetes. The period for recurrence was 1 to 3 months in seven patients (47\%), but was up to $\geq 3$ years in four patients (27\%).

\section{Discussion}

Peritonsillar abscess is often reported to occur in males in their early to middle adulthood. ${ }^{1,3-7}$ Our study was consistent with the previous reports, as males accounted for $66 \%$ of the patients in our study; also, majority of them were in their $30 \mathrm{~s}$ to $40 \mathrm{~s}$. The higher rates of male patients with this condition can be attributed to the antiplasminogenic effects and anti-inflammatory properties of the female hormone estrogen. $^{8}$

There were no differences in the affected side, although many studies report 0 to $3 \%$ bilateral abscesses. ${ }^{3,6}$ The abscesses are localized in the superior pole more frequently than in the inferior pole; the results of our study were similar to the results of previous studies in this regard. Greater depth and proximity to the capsule and infections of Weber's gland located anterosuperiorly are some of the proposed reasons for the higher incidence of abscesses in the superior pole. ${ }^{1}$

Laryngeal edema is a frequent complication of peritonsillar abscesses of the inferior pole. Indeed, $61 \%$ of lower pole abscesses were complicated with laryngeal edema, and all cases that required a tracheostomy were localized in the inferior pole. However, $12.5 \%$ of superior pole abscess types are also complicated with laryngeal edema, ${ }^{4}$ as was observed in 13 patients with superior pole abscess (18\%). In this investigation, cases of inferior pole abscesses had higher CRP levels in blood samples taken on admission and significantly higher rates of laryngeal edema than cases of superior pole abscesses. This is because the inferior tonsillar pole has a more fragile external wall than the superior pole, and the peritonsillar space is closed by the pharyngeal constrictor muscle, which tends to make laryngeal symptoms appear earlier and spread inflammation more easily. ${ }^{9,10}$

When symptoms like peritonsillar swelling or uvular displacement typical in inferior pole abscesses are absent, or when the site is difficult to access for incisional drainage, progression to laryngeal edema can be rapid, ${ }^{11}$ entailing the need for caution.

In our department, incisional drainage is performed for treatment as much as possible. Steroid therapy is effective for treating early subjective symptoms, eating, and reducing 


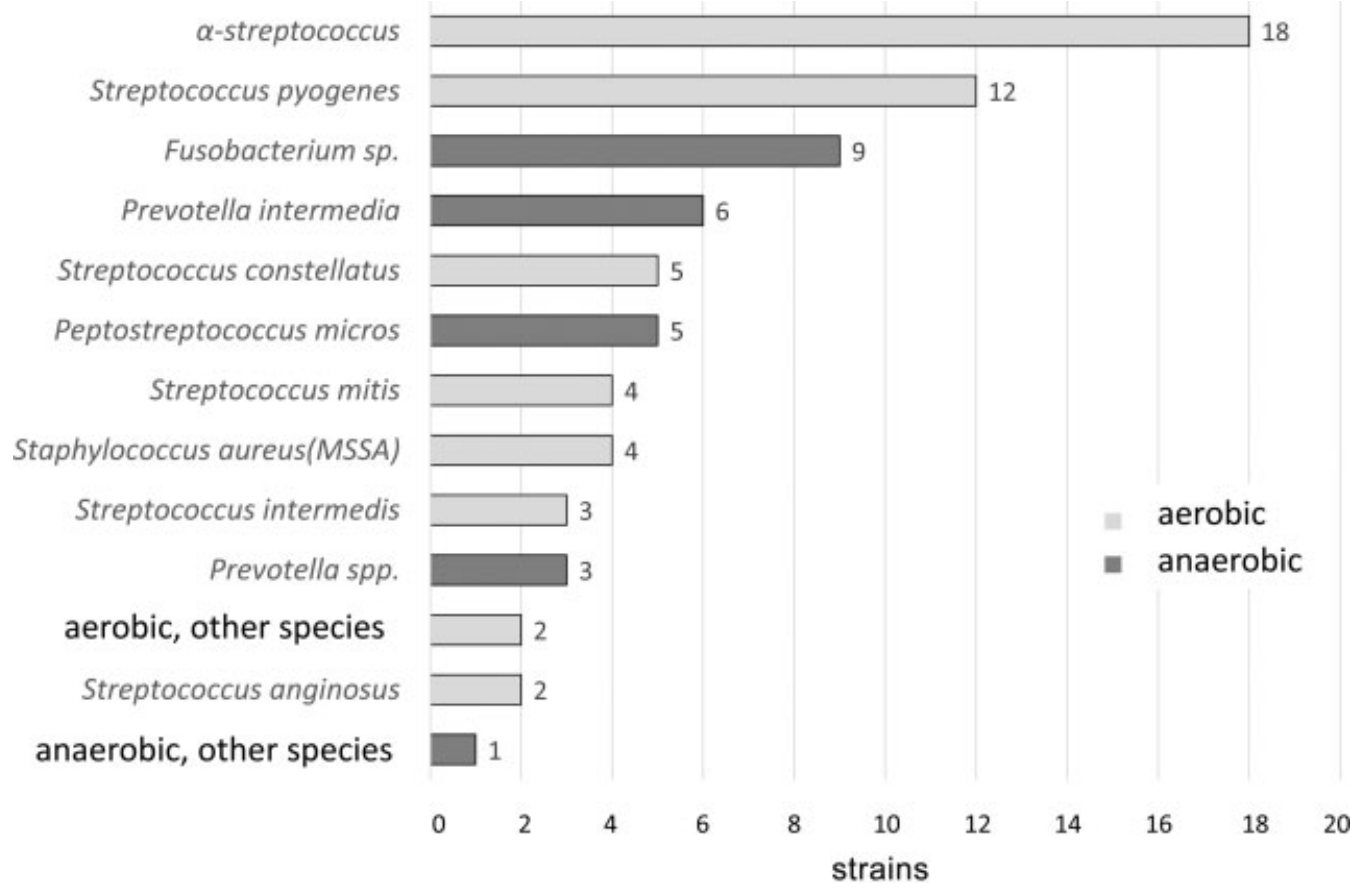

Fig. 6 Prevalence of detected bacterial strains. Among the aerobic strains, $\alpha$-streptococcus ( 18 strains) was the most numerous, and among the anaerobic strains, Fusobacterium (9 strains) was the most numerous.

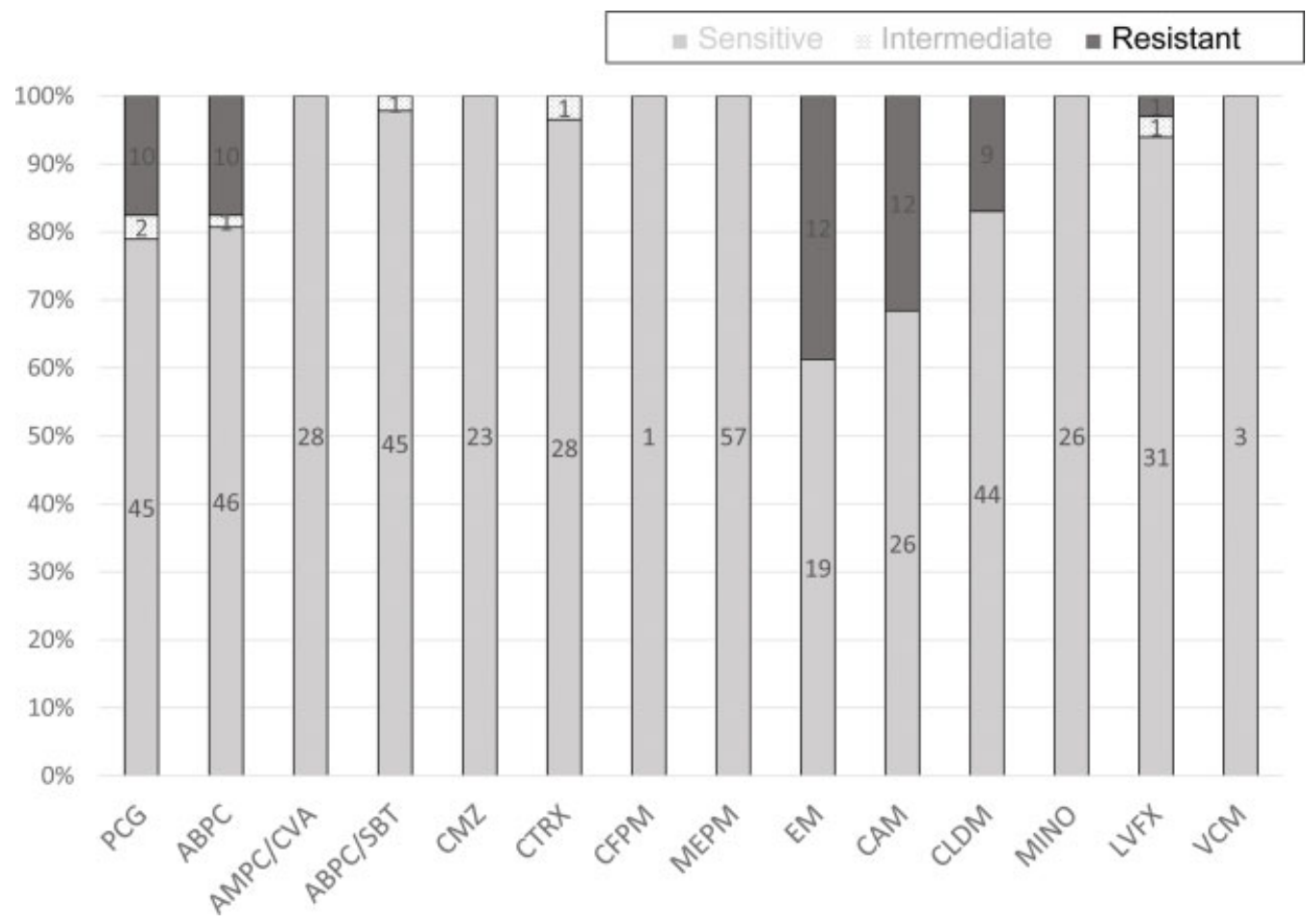

Fig. 7 Drug sensitivity and resistance according to antibiotic type. Resistant strains were resistant to CAM, EM, PCG, ABPC, CLDM, and LVFX. $A B P C$, ampicillin; $A B P C / S B T$, ampicillin/sulbactam; AMPC/CVA, amoxicillin/clavulanate; CAM, clarithromycin; CFPM, cefepime; CLDM, clindamycin; CMZ, cefmetazole; CTRX, ceftriaxone; EM, erythromycin; LVFX, levofloxacin; MEPM, meropenem; MINO, minocycline; PCG, benzylpenicillin; VCM, vancomycin.

hospital stay. ${ }^{12}$ Hence, steroids are also administered in cases that are severe and those involving laryngeal edema. It was administered in $40 \%$ patients in this study; however, significantly shorter hospital stays were not achieved. Nonetheless, considering that steroids were only administered to severe cases, we can interpret that the lengths of hospital stays were shortened to those more typical of mild cases.

According to one report, steroid use does not result in a difference in recurrence rate. ${ }^{13}$ Hence, steroid should be administered for short periods to shorten disease duration 
for abscesses of the inferior pole or abscesses involving intense pharyngalgia, considering comorbidities such as diabetes.

Anaerobic bacteria have been isolated in approximately 30 of $60 \%$ cases, depending on facilities. ${ }^{3,6,14}$ Similar to previous studies, bacteria were detected in $36 \%$, including mixed infections. Reports of CLDM-resistant bacterial strains have increased recently, ${ }^{3,14-16}$ and our investigation also detected $17 \%$ strains that were CLDM-resistant. None of the strains were resistant to $A B P C / S B T$, suggesting good therapeutic effects of ABPC/SBT monotherapy. In our department, $\mathrm{ABPC} / \mathrm{SBT}$ is used as the first-line therapy. Although it is considered an appropriate choice of antibiotic, samples should be cultured as much as possible to select antibiotics based on the knowledge of drug sensitivity to prevent emergence of resistant strains in the future.

Recurrences have been reported to range between 3 and $24.7 \%$ in previous reports., ${ }^{3,5,17}$ In our study, recurrences occurred in $14 \%$ cases, which were somewhat more frequent in younger patients. Presence or absence of comorbidities, such as diabetes, investigated in our study did not have any correlation with recurrence. Also, $47 \%$ of recurrent cases occurred within 3 months. A previous study reported that recurrence occurred after $\geq 1$ year in $40 \%$ of the recurrent cases. ${ }^{3}$ Among the patients in our study, seven had a recurrence after $>1$ year (47\%), of which four had a recurrent abscess after $>3$ years (27\%), suggesting the importance of informing patients about the possibility of recurrences occurring after long time periods.

\section{Summary}

- We retrospectively investigated 107 cases of peritonsillar abscess that required hospitalization for treatment at our department in the past 5 years.

- Peritonsillar abscesses occurred commonly in males in their 30s to 40s. Laryngeal edema was a complication that occurred in many cases of inferior pole abscesses, but also in $18 \%$ cases of superior pole abscesses. The three patients that required airway maintenance were cases of inferior lobe abscesses.

- No strains resistant to ABPC/SBT were detected, suggesting good therapeutic effects of ABPC/SBT monotherapy.

- Recurrences occurred in 15 patients (14\%). The recurrence occurred in $<3$ months in seven cases ( $47 \%$ ) and $\geq 3$ years in four cases (27\%), suggesting the importance of informing patients about the possibility of recurrences after long time periods.
Conflicts of Interest

None declared.

\section{References}

1 Kawabata M, Umakoshi M, Matsumoto $\mathrm{H}$, et al. The clinical characteristics of inferior pole peritonsillar abscesses. Stomatopharyngology 2018;31:187-192

2 Katori H, Tsukuda M. Acute epiglottitis: analysis of factors associated with airway intervention. J Laryngol Otol 2005;119(12): 967-972

3 Saiki T, Sakaki M, Watanabe F. Clinical study of 215 cases of peritonsillar abscess. Practica Oto-Rhino-Laryngologica 2010; 11:1021-1027

4 Hara H, Tarumoto S, Sugawara K, et al. A clinical study of peritonsillar abscess with laryngeal edema. J Jpn Soc Infect Dis Otolaryngol 2011;29:37-40

5 Kurata N, Ishihara A, Sakamoto M, et al. A study on peritonsillar abscesses: severity of peritonsillar abscess and ambulatory care [in Japanese]. Pract Otorhinolaryngol (Basel) 2014;107:993-1001

6 Kato H, Wada I, Nakada H, et al. A clinical and statistical study of admitted peritonsillar abscess cases [in Japanese]. Stomato-pharyngology 2006;18:421-428

7 Iida S, Murata K, Tamaki K, et al. Peritonsillar abscess: a review of 64 patients. Practica Oto-Rhino-Laryngologica 1989;82: 1101-1107

8 Kraitrakul S, Sirithunyaporn S, Yimtae K. Distribution of minor salivary glands in the peritonsillar space. J Med Assoc Thai 2001; 84(03):371-378

9 Ohori J, Umakoshi M, Miyashita K, Hayamizu Y, Harada M, Kurono Y. Computed tomography image analysis of peritonsillar abscess [in Japanese]. Nippon Jibiinkoka Gakkai Kaiho 2013;116(08): 947-952

10 Licameli GR, Grillone GA. Inferior pole peritonsillar abscess. Otolaryngol Head Neck Surg 1998;118(01):95-99

11 Nishimoto K, Ohori J, Hayamizu Y, et al. Clinical aspects of superior and inferior pole peritonsillar abscess. J Jpn Soc Infect Dis Otolaryngol 2006;24:105-108

12 Koçak HE, Acıpayam H, Elbistanlı MS, et al. Is corticosteroid a treatment choice for the management of peritonsillar abscess? Auris Nasus Larynx 2018;45(02):291-294

13 de Cassan S, Thompson MJ, Perera R, et al. Corticosteroids as standalone or add-on treatment for sore throat. Cochrane Database Syst Rev 2020;5:CD008268

14 Umibe A, Anazawa U, Kessoku H, et al. Clinical analyses of 115 patients with peritonsillar abscess [in Japanese]. Nippon Jibiinkoka Gakkai Kaiho 2015;118(10):1220-1225

15 Hasegawa $\mathrm{H}$, Ito $\mathrm{M}$, Yoshizaki $\mathrm{T}$, et al. Optimal antibiotics for treating peritonsillar abscess [in Japanese]. Practica Oto-RhinoLaryngologica 2013;106:609-613

16 Adachi M, Tobita T, Akizuki H. A Study on 140 hospitalized patients with peritonsillar abscess [in Japanese]. Practica OtoRhino-Laryngologica 2019;112:47-53

17 Ueyama T, Suzuki M, Shigemi H, et al. Clinical study of patients with peritonsillar abscess. J Jpn Soc Infect Dis Otolaryngol 1998; $16: 117-120$ 